\title{
Bilateral advancement technique for laryngectomy stomaplasty
}

Thomas Jacques, Lenka Adamová, Zaid Awad Imperial College Healthcare NHS Trust, London

\section{Background}

The maintenance of a suitably-sized stoma is key in rehabilitating laryngectomy patients. Inadequate stoma size can arise due to surgical technique, excessive granulation or delayed shrinkage. Stomaplasty has no single widely-adopted technique.

Inadequate stomal size can arise due to surgical technique, excessive granulation or delayed shrinkage.

\section{Surgical technique}

- Bilateral broad-based trapezoid subplatysmal flaps are raised, incorporating lateral stomal skin-mucosal junctions.

- Cartilaginous scar ring excised; scar debridement and/or sternomastoid tenotomy can be performed.

- Superior portion of the stoma is left undisturbed, to avoid impact on voice prosthesis position and repeat circumferential injury.

- Trachea is mobilised if needed to reduce tension.

- Flaps are advanced to the new stomal margin and secured with PDS halfmattress sutures to cover all exposed cartilage.
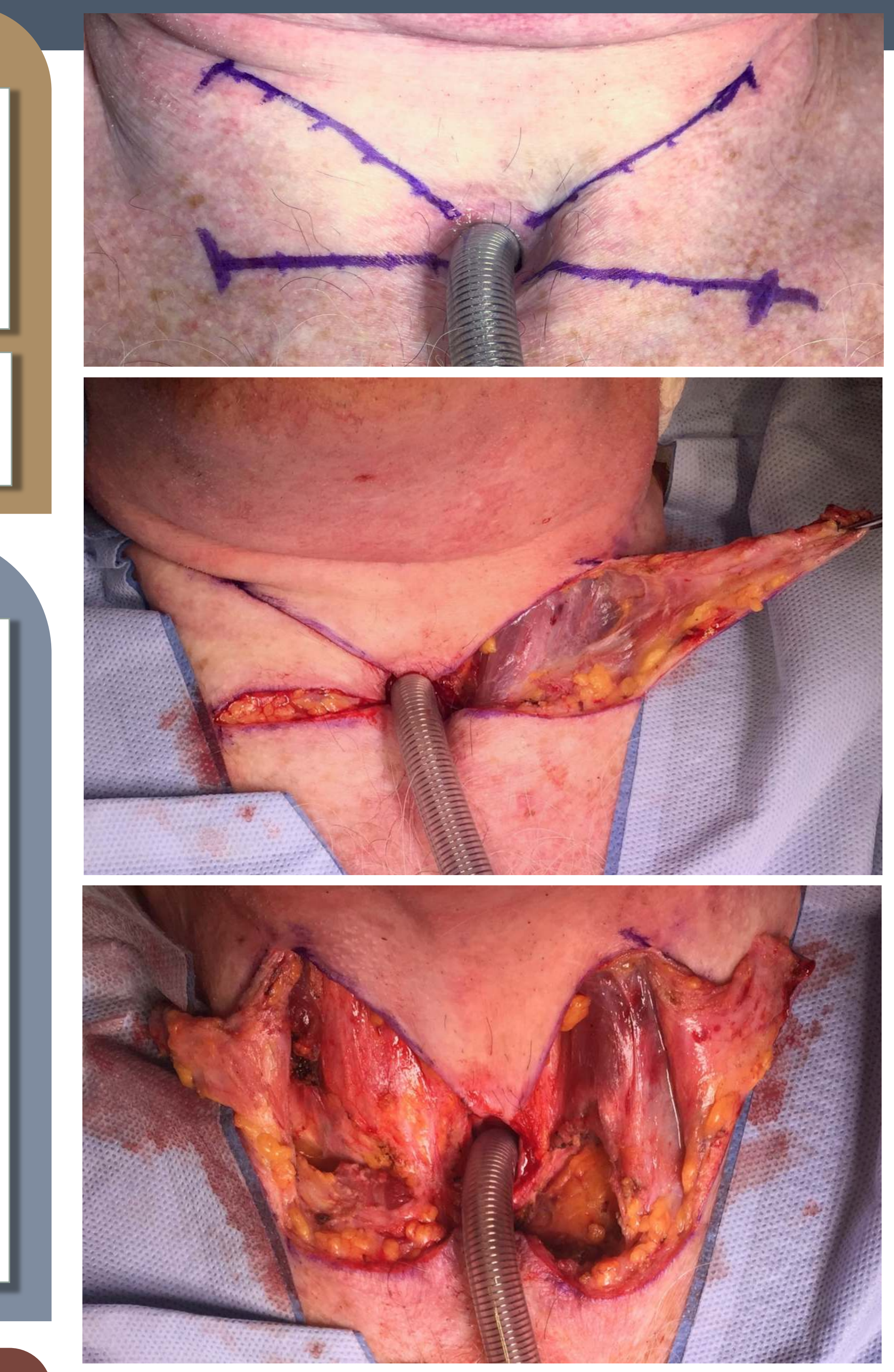

\section{Postoperative care}

Laryngectomy tube, ointment, antibiotics, regular de-crusting, suture removal in two weeks.

\section{Conclusion}

This surgical technique allows straightforward, reproducible enlargement of the small laryngectomy stoma. The broad pedicle allows robust tissue advancement in irradiated patients.

Background
The maintenance of a suitably-sized stoma
is key in rehabilitating laryngectomy patients.
Inadequate stoma size can arise due to
surgical technique, excessive granulation or
delayed shrinkage. Stomaplasty has no
single widely-adopted technique.
Inadequate stomal size can arise due to
surgical technique, excessive granulation or
delayed shrinkage.

Surgical technique
- Bilateral broad-based trapezoid
subplatysmal flaps are raised,
incorporating lateral stomal skin-mucosal
junctions.
- Cartilaginous scar ring excised; scar
debridement and/or sternomastoid
tenotomy can be performed.
- Superior portion of the stoma is left
undisturbed, to avoid impact on voice
prosthesis position and repeat
circumferential injury.
- Trachea is mobilised if needed to reduce
tension.
- Flaps are advanced to the new stomal
margin and secured with PDS half-
mattress sutures to cover all exposed
cartilage.

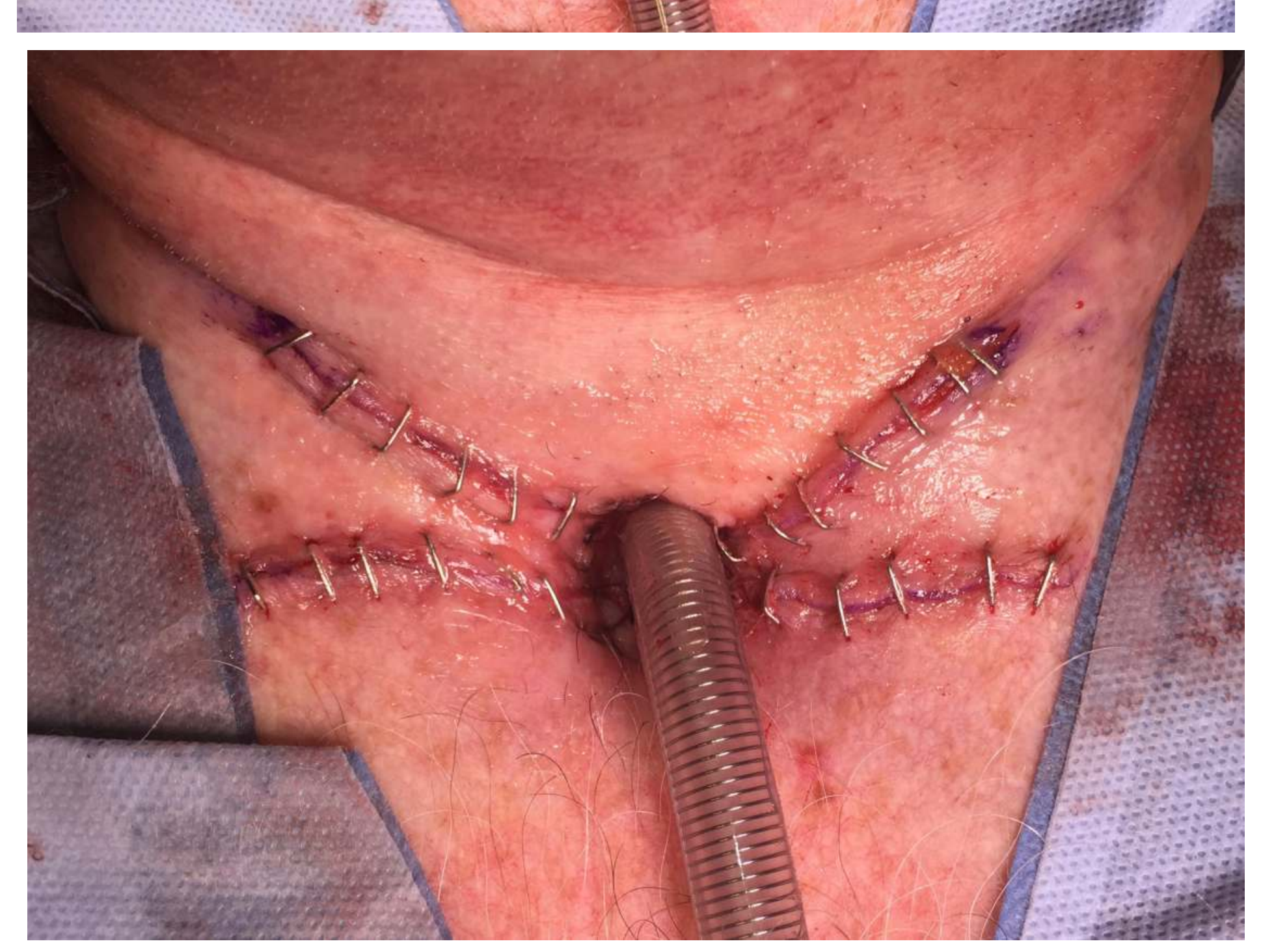

